\title{
The effect of myometrial invasion on prognostic factors and survival analysis in endometrial carcinoma
}

\author{
Cem Dane, Sait Bakir
}

University of Health Sciences, Haseki Training and Research Hospital, Department of Gynecology and Obstetrics, Istanbul-Turkey.

\begin{abstract}
Background: We investigated the relationship between myometrial invasion and the prognostic factors on overall and progression free survival in endometrial carcinoma.

Methods: 122 cases operated with endometrial cancer were included into the study. Progression-free survival and overall survival were evaluated according to degree of myometrial invasion. We also investigated the relationship between myometrial invasion and prognostic factors.

Results: The 5- year progression-free survival rate was $90 \%$ in stage I, $66 \%$ in stage II, $32 \%$ in stage III and $60 \%$ in stage IV. The 5- year overall survival rate was $95 \%$ in stage I, $89 \%$ in stage II, $49 \%$ in stage III and $30 \%$ in stage IV. The progression free survival and overall survival for patients with more than $50 \%$ myometrial invasion were detected $67 \%$ at 58 months and $66 \%$ at 60 months, respectively. The clinicopathological variables that significantly correlated with myometrial invasion of more than $50 \%$ were as follows: pelvic lymph node metastasis (p: 0,00029-OR: 11.2), cervical stromal invasion (p: 0008-OR:7.9), LVSI $(\mathrm{p}<0.0001-\mathrm{OR}: 16.5)$.

Conclusion: The depth of myometrial invasion is one of the most important prognostic indicators and determinants of therapy in endometrial cancer.

Keywords: Endometrial carcinoma; Progression free survival; Overall survival; Prognostic factors.

DOI: https://dx.doi.org/10.4314/ahs.v19i4.47

Cite as: Dane C, Bakir $S$. The effect of myometrial invasion on prognostic factors and survival analysis in endometrial carcinoma. Afri Health Sci.2019;19(4):3235-3241. https:/ / dx.doi.org/10.4314/abs.v19i4.47
\end{abstract}

\section{Introduction}

Endometrial cancer affects mainly postmenopausal women. The average age of women diagnosed with endometrial cancer is 60 . It is uncommon in women under the age of 45 . In 2018, it is estimated that there will be 63,230 new cases of uterine cancer and an estimated 11,350 people will die of this disease ${ }^{1}$. These estimates include both endometrial cancers and uterine sarcomas. About 63,230 new cases of cancer of the uterine corpus will be diagnosed, but only about 5,058 of these cases will be uterine

\section{Corresponding author:}

\section{Cem Dane,}

University of Health Sciences,

Haseki Training and Research Hospital,

Department of Gynecology and Obstetrics,

Division of Gynecologic Oncology, Istanbul-Turkey

Tel: 902126217521 Fax: 902125896229

Email: cemdane@yahoo.com sarcomas. Up to $8 \%$ of uterine corpus cancers are sarcomas, so the actual numbers for endometrial cancer cases and deaths are slightly lower than these estimates ${ }^{2}$. Fortunately, the majority of women with endometrial cancer are diagnosed at an early stage (the disease is confined to the uterine corpus) and may be cured by surgery with or without adjuvant radiotherapy ${ }^{3}$. Survival is obviously stage related. According to a study by Creasman; the 5year progression-free survival rate was $92 \%$ in stage I, 82 $\%$ in stage II, $68 \%$ in stage III and $29 \%$ in stage IV4. The 5- year overall survival rate was $89 \%$ in stage I, $78 \%$ in stage II, $61 \%$ in stage III and $21 \%$ in stage IV $^{4}$.

In endometrial carcinoma, myometrial invasion is a wellknown predictor of recurrence, and important in the decision making for adjuvant treatment ${ }^{5}$.

The objective of this study was to analyze the effect of myometrial invasion on overall and progression free survival in endometrial carcinoma. The relationship between myometrial invasion and other known prognostic factors in endometrial cancer was also studied. 


\section{Material and methods}

We reviewed the records of 122 endometrial carcinoma patients treated at University of Health Sciences, Haseki Training and Research Hospital, Department of Gynecology and Obstetrics, Division of Gynecologic Oncology. Ethics Committee Approval was taken from the local committee of our institution. All patients underwent staging procedures including total hysterectomy, adnexectomy, peritoneal washings for cytology, pelvic and para-aortic lymphadenectomy, and omentectomy if indicated. All cases were histologically confirmed as endometrioid adenocarcinoma, papillary serous carcinma, clear cell carcinoma, or carcinosarcoma, and were included in the study. All cases of endometrial stromal sarcoma and leiomyosarcoma were excluded. Progression-free survival (PFS) was defined as the period between the time of surgery and the observation of the recurrence. Overall survival (OS) was the time between the surgery and death, and follow-up time was evaluated as the time between the surgery and the time that the patient was last examined (death or last visit).

\section{Statistical analysis}

Data were analyzed using SPSS statistical software, version 11.5. Descriptive statistics were used to analyze demographic data and were summarized as numbers with percentage or median with range. Progression free survival (PFS), overall survival (OS) were analyzed by the Kaplan-Meier method. Comparisons of survival curves were performed using the log-rank test. The effect of myometrial invasion was analyzed taking into account other variables significantly linked to survival. Odds ratios and $95 \%$ confidential intervals were used to assess the risk of myometrial invasion in relation to each pathological variable. $\mathrm{P}$-values of $<0.05$ were considered significant.

\section{Results}

A total of 122 patients with endometrial cancer under- went primary surgery at our institution. The average age of patients was $58.5 \pm 10$ years (median 58; range $32-86$ years. All the patients underwent abdominal hysterectomy and bilateral salpingo-oophorectomy (BSO). 109 patients underwent a hysterectomy and bilateral salpingo-oophorectomy with pelvic lymphadenectomy. Pelvic and/or para-aortic lymphadenectomy was performed in 19 patients. Omentectomy was performed in 102 patients. Intraoperative peritoneal cytology was performed in $122 \mathrm{pa}$ tients. At the time of diagnosis, 26 patients $(14.8 \%)$ were pre-menopausal, and $96(85.2 \%)$ were postmenopausal. Patient's characteristics

Endometriod adenocarcinoma was the most common histological form of endometrial cancer. Histologically, while $112(91.8 \%)$ patients were endometrioid, the remaining $10(8.1 \%)$ had non-endometrioid histology. Histological grades were as follows: $59(48.3 \%)$ grade 1, 38 $(31.1 \%)$ grade 2 , and $25(20.4 \%)$ grade 3 . The distribution of surgical FIGO stages were 77 (63.1\%) Stage Ia, $21(17.2 \%)$ Stage Ib, 8 (6.5\%) Stage II, 1 (0.8\%) Stage IIIa, 1 (0.8 \%) Stage IIIb, 7 (5.7 \%) Stage IIIc1, 2 (1.6\%) Stage IIIc2, 1 (0.8\%) Stage IVa, and 4 (3.2\%) Stage IVb. Myometrial invasion was documented in 97 cases $(79.5$ $\%$ ). Myometrial invasion was found $74.4 \%$ in stage I cases. Adnexal spread was detected in $5.7 \%$, it's was found 2 cases in stage 3 and all cases in stage 4 . Cervical involvement was seen in $18 \%$ of the patients. These patients are evaluated as separate $4 \%$ in stage I and $6.5 \%$ in stage II, Stage III and Stage IV at $4 \%, 3.2 \%$, respectively. Lymphovascular space invasion was detected in 32 (26.2\%) cases. LVSI evaluation of our patients when the absence of invasion in 90 patients $(73.7 \%) 32$ patients were found to be in the invasion $(26.2 \%)$. When analyzed LVSI according to the stage $12.2 \%$ in stage I, $50 \%$ in stage II, $100 \%$ in Stage III and Stage IV. Of the 122 subjects, 9 patients $(7.4 \%)$ had positive peritoneal cytology and 113 $(92.6 \%)$ had negative cytology. Four of them were in stage I, 1 in stage II, in 1 case in stage III and in stage IV in 3 cases. Omental metastases were found in 4 (3.9\%) of 102 patients. 
Table 1- The relationship between $>50 \%$ myometrial invasion - prognostic factors

\begin{tabular}{|l|l|l|l|}
\hline Parameters & Odds ratios & $\begin{array}{l}95 \% \text { Confidential } \\
\text { intervals }\end{array}$ & P values \\
\hline $\begin{array}{l}\text { Myometrial Invasion- } \\
\text { Pelvic Lymph Node } \\
\text { Involvement }\end{array}$ & 11.2 & $2,2-55,3$ & 0,00029 \\
\hline $\begin{array}{l}\text { Myometrial Invasion - } \\
\text { Cervical } \\
\text { Involvement }\end{array}$ & 7.9 & $2,3-26,6$ & 0,0008 \\
\hline $\begin{array}{l}\text { Myometrial Invasion - } \\
\text { Omental Involvement }\end{array}$ & 16.8 & $0,8-321,7$ & $0,06 *$ \\
\hline $\begin{array}{l}\text { Myometrial Invasion - } \\
\text { Lympho Vascular Space } \\
\text { Involvement }\end{array}$ & 16.5 & $6,09-44,7$ & 0,0001 \\
\hline $\begin{array}{l}\text { Myometrial Invasion - } \\
\text { Adnexal spread }\end{array}$ & 35.4 & $1,9-637,7$ & 0,001 \\
\hline $\begin{array}{l}\text { Myometrial Invasion - } \\
\text { Peritoneal cytology }\end{array}$ & 16.4 & & $0,139,0$ \\
\hline
\end{tabular}

*P $>0.05$ insignificantly.

Pelvic lymph node dissection was performed on a hundred-nine patients and both pelvic and para-aortic lymph node dissection was performed on nineteen patients. In total, 14 patients $(11.4 \%)$ had lymph node metastases. Eleven patients (n: $11 / 109,10 \%$ ) had metastasis in the pelvic region, and three patients (n: 3/19, $15.7 \%$ ) had tumor in the para-aortic region. Seventeen patients (13.9 $\%$ ) developed recurrences.

\section{Recurrences}

The recurrence was detected 17 patients. Recurrence was outside of the pelvic region in 6 patients. The recurrence site was vaginal in 11 patients $(64.7 \%)$, abdominal skin in 1 patient $(5.8 \%)$, liver in 3 patients $(17.6 \%)$, and small intestine in 2 patients $(11.7 \%)$. The recurrence rate was detected $37.5 \%$ excluded Stage Ia cases. 77 patients were not included in the analysis because of Stage Ia.

\section{Survival analysis}

Kaplan-Meier survival analysis was performed in the evaluation of 122 patients. The 5-year PFS of the patients according to stage of the disease was: $90 \%$ in stage I, $66 \%$ in stage II, $32 \%$ in stage III and $60 \%$ in stage IV. Between the survival curves were significantly difference in Kaplan-Meier analysis $(\mathrm{p}<0,0001)$. The OS of the $\mathrm{pa}-$ tients according to stage of the disease was: $95 \%$ in stage I, $89 \%$ in stage II, $49 \%$ in stage III and $39 \%$ in stage IV. Between the survival curves were significantly difference in Kaplan-Meier analysis $(\mathrm{p}<0,0001)$ (Figure 1). 

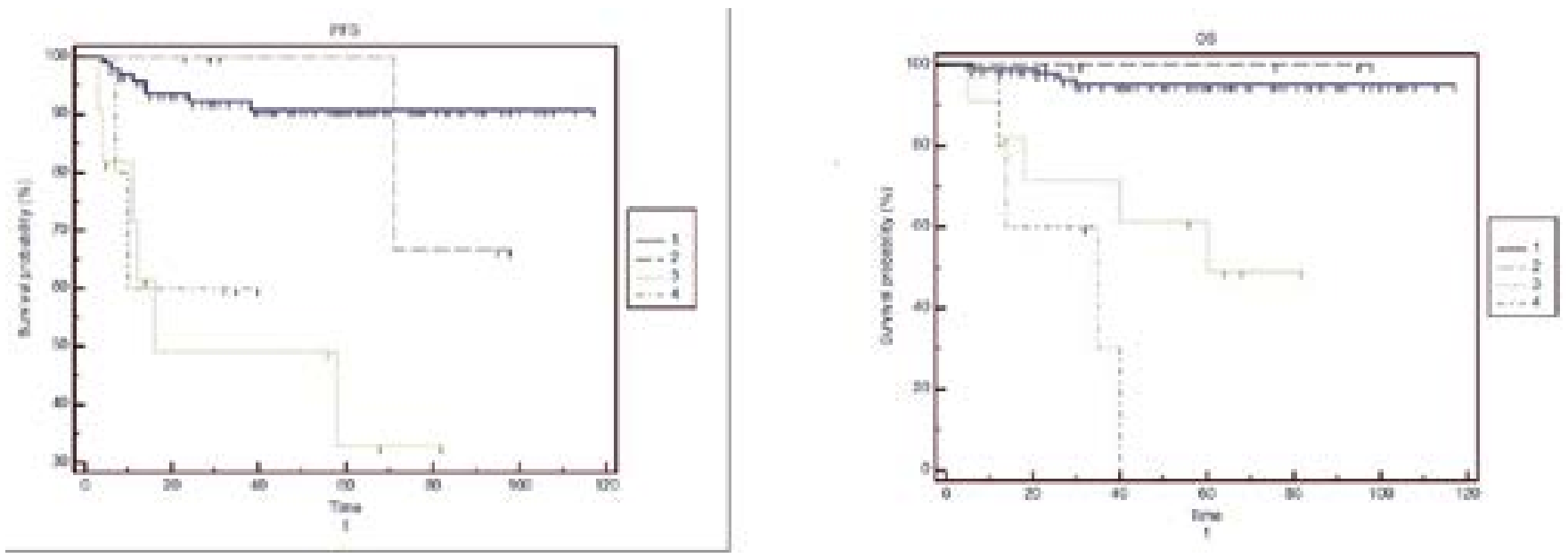

Figure 1- Progression-free survival and overall survival in the study population.

The 5-year PFS rate for patients with myometrial invasion $<50 \%$ was $88 \%$ compared $67 \%$ for the patients with myometrial invasion $>50 \%$ (p: 0,0013). The 5-year OS rate was $96 \%$ for patients with myometrial invasion $<50$
$\%$ versus $66 \%$ for those with myometrial invasion $>50$ $\%(\mathrm{p}<0,0001)$. Between the survival curves were significantly difference in Kaplan-Meier analysis both PFS and OS (Figure 2).
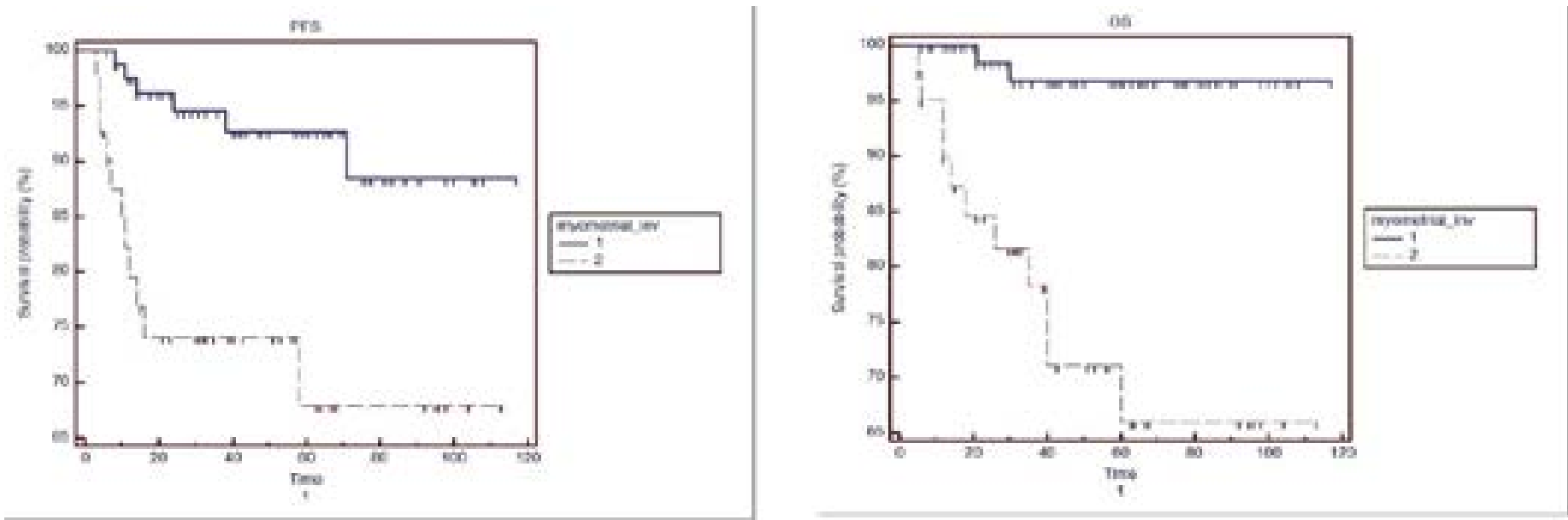

Figure 2- Progression-free survival and overall survival at $>50 \%$ myometrial invasion.

The relationship between myometrial invasion prognostic factors

When patients with myometrial invasion of more than 50 $\%$, pelvic lymph node metastasis, cervical involvement, LVSI, adnexal spread, peritoneal cytology positivity, and omental involvement were analyzed (Table-1). Statistically significant associations were found between deep myometrial invasion and cervical stromal invasion (OR:
7,9- 95\% CI: 2,3-26,6- p: 0,0008), positive peritoneal fluid cytology (OR: 16,4-95\% CI: 1,9-139,0-p: 0,01), adnexal spread (OR: 35,4-95\% CI: 1,9-637,7-p: 0,001), LVSI (OR: 16,5-95\% CI: 6,09- 44,7-p<0,0001), and pelvic lymph node involvement (OR: 11.2-95\% CI: 2.2-55,3-p: $0,00029)$. No association was found between myometrial invasion and the omental metastases (OR: 16,8-95\% CI: 0,8-321,7-p: 0,06). 


\section{Discussion}

In this study, we evaluated the relationship between myometrial invasion and prognostic factors- survival analysis. Myometrial invasion was documented in 97 cases $(79.5$ $\%$ ). Myometrial invasion was found $74.4 \%$ in stage I cases. In the present study, the progression free survival and overall survival for patients with more than $50 \%$ myometrial invasion were detected $67 \%$ and $66 \%$, respectively. Recently, screening of biological indexes and gene therapies for endometrial carcinoma is becoming an active research field. Specific indicators of adverse outcomes are needed in order to tailor the management of endometrial cancer and improve their survival rates. During the last decades microcystic, elongated, fragmented (MELF) histologic pattern has been examined and proposed as a prognostic marker in patients with endometrial carcino$\mathrm{ma}^{6}$. Murray et al firstly described the MELF pattern of invasion in patients with endometrial adenocarcinoma ${ }^{7}$. Significantly higher rates of myometrial invasion $>50 \%$ were detected in patients with MELF compared to those with non-MELF ${ }^{8}$. Another study from Sanci et al reported significant impact on overall survival (OS) n patients with MELF pattern compared with those with MELF $(-)^{9}$.

Tripartite motif containing 44 (TRIM44) has been demonstrated to be important in tumor metastasis and progression. High TRIM44 expression was associated with poor overall survival (OS) or disease-free survival (DFS) in EC patients ${ }^{10}$. High TRIM44 protein expression was significantly correlated with the FIGO stage, depth of myometrial invasion, histological grade and lymph node metastasis ${ }^{10}$.

Myometrial invasion depth and lymph node involvement are also known to affect survival in endometrial carcinoma. In a large study carried out by Jones et al. five years' survival rates of patients with or without deep myometrial invasion were found as $60 \%$ and $80 \%$, respectively ${ }^{11}$. Five years' survival rates of deep myometrial invasion in the literature were $71-88 \%$ for grade I, $60-79 \%$ for grade II and 32-65 \% for grade III ${ }^{12,13}$. In the present study, the patients with myometrial invasion $<50 \%$ was $88 \%$ compared $67 \%$ for the patients with myometrial invasion $>50 \%$ at 5 year PFS (p:0,0013). The 5-year OS rate was $96 \%$ for patients with myometrial invasion $<50$ $\%$ versus $66 \%$ for those with myometrial invasion $>50$ $\%(\mathrm{p}<0,0001)$. We found a significant difference between PFS rates of patients with and without myometrial invasion.
According to the prognostic parameters for endometrial carcinoma of the uterine factors include histological type, histological grade and depth of myometrial invasion, lymphovascular invasion, presence of atypical endometrial hyperplasia, cervical involvement, DNA ploidy and S-shape fraction and hormone receptor status. The extra-uterine factors include positive peritoneal cytology, adnexal involvement, pelvic and para-aortic lymph node metastasis and peritoneal metastasis ${ }^{14,15}$. For a patient with endometrial cancer, the presence of deep myometrial invasion is an important prognostic factor that strongly affects treatment planning and prognosis ${ }^{5}$. In this study, the prognostic value and relationship with clinicopathologic parameters of myometrial invasion were analyzed and compared.

The depth of myometrial invasion is a highly important factor that can be used to predict nodal metastasis ${ }^{16}$. Because of the higher prevalence of lymph node metastases, patients with depth of myometrial invasion are more likely to have a more extensive surgical lymph node assessment than patients with absence of myometrial invasion $^{17}$. In present study, we found pelvic lymph node and para-aortic lymph node metastasis, $9 \%, 2.4 \%$, respectively.

The patients with more than $50 \%$ myometrial invasion on gross intraoperative examination of the uterus appear to have a six- to seven-fold higher prevalence of pelvic lymph node metastases, and advanced surgical stage compared with women with less than 50\% invasion, patients with deep myometrial invasion on gross examination should be considered for more aggressive surgical staging, including pelvic and para-aortic lymphadenectomy ${ }^{18}$. Invasion to more than half of the myometrium in patients were significantly associated with cervical extension, positive peritoneal fluid cytology, adnexal, LVSI and pelvic lymph node involvement in present study. However, there was no statistically significant association between omental involvement.

Previous studies showed that the tumor grade and the depth of myometrial invasion, as well as LVSI, are useful predictors for the risk of lymph node metastasis ${ }^{12,19}$. The incidence of LVSI in patients with endometrial cancer is reported to be about $25 \%$ when all the stages and tumor grades are included20. In the present study, LVSI was positive in 32 patients (26.2 \%), and this was similar to the results of previous studies. 
Patients with more than $50 \%$ gross myometrial invasion have a 6.4-fold higher prevalence of pelvic lymph node metastases and a 6.9-fold higher prevalence of para-aortic lymph node metastases than patients with less than 50 $\%$ myometrial invasion ${ }^{21}$. Creasman et al., reported that the incidence of para-aortic lymph nodal metastases increased from $2 \%$ in patients with no myometrial invasion to $21 \%$ with deep myometrial invasion ${ }^{12}$. In our study, statistically significant associations were found between deep myometrial invasion and pelvic lymph node involvement (OR: 11.2, p: 0,00029).

In the study of Ayhan et al., there were extra-abdominal metastases in $16 \%$; liver, spleen, or diaphragmatic spread in $24 \%$; small intestine involvement in $24 \%$; and large intestine involvement in $19 \%$ of the patients 22 . The recurrence rate in our present study was $37.5 \%$ (17 patients) excluded stage I. The recurrence site was vaginal in 11 patients $(64.7 \%)$, abdominal skin in 1 patient (5.8 $\%$ ), liver in 3 patients $(17.6 \%)$, and small intestine in 2 patients $(11.7 \%)$.

This study had several limitations. The sample size was relatively small and the study was retrospective in nature. Despite this limitation, the primary strength of the study was that it was conducted in a single institution, where the pathological specimens are reviewed by experienced gynecologic pathologists. The size of our study cohort was sufficient for analyzing the prognostic factors that were independently associated with DFS and OS.

\section{Conclusion}

This single-center retrospective analysis confirms that myometrial invasion are important prognostic factors of progression-free survival and overall survival in patients diagnosed with endometrial cancer.

\section{Conflict of interest}

None declared.

\section{References}

1. Siegel RL, Miller KD, Jemal A. Cancer statistics, 2018. CA Cancer J Clin 2018; 68: 7-30.

2. Koh WJ, Abu-Rustum NR, Bean S, Bradley K, Campos SM, Cho KR. Uterine Neoplasms, Version 1.2018, NCCN Clinical Practice Guidelines in Oncology. J Natl Compr Canc Netw. 2018; 16:170-99.

3. Korcum AF, Duman E, Aksu G. The results of adjuvant radiotherapy in endometrial carcinoma. Gynecol Endocrinol. 2010; 26: 240-5.
4. Creasman WT, Odicino F, Maisonneuve P, Quinn MA, Beller U, Benedet JL. Carcinoma of the corpus uteri. FIGO 26th Annual Report on the Results of Treatment in Gynecological Cancer. Int J Gynaecol Obstet. 2006; 95 Suppl 1:S105-43.

5. Geels YP, Pijnenborg JM, van den Berg-van Erp SH, Snijders MP, Bulten J, Massuger LF. Absolute depth of myometrial invasion in endometrial cancer is superior to the currently used cut-off value of 50\%. Gynecol Oncol. 2013; 129: 285-91.

6. Prodromidou A, Vorgias G, Bakogiannis K, Kalinoglou $\mathrm{N}$, Iavazzo C. MELF pattern of myometrial invasion and role in possible endometrial cancer diagnostic pathway: A systematic review of the literature. Eur J Obstet Gynecol Reprod Biol. 2018; 230: 147-52.

7. Murray SK, Young RH, Scully RE. Unusual epithelial and stromal changes in myoinvasive endometrioid adenocarcinoma: a study of their frequency associated diagnostic problems, and prognostic significance. Int J Gynecol Pathol 2003; 22: 324-33.

8. Kihara A, Yoshida H, Watanabe R, Takahashi K, Kato T, Ino Y. Clinicopathologic Association and Prognostic Value of Microcystic, Elongated, and Fragmented (MELF) Pattern in Endometrial Endometrioid Carcinoma. Am J Surg Pathol. 2017; 41: 896-905.

9. Sanci M, Güngördük K, Gülseren V, Karadeniz T, Kocaer M, Gungorduk O. MELF Pattern for Predicting Lymph Node Involvement and Survival in Grade I-II Endometrioid-type Endometrial Cancer. Int J Gynecol Pathol. 2018 ; 37: 17-21.

10. Li P, Yin H, Meng F, Liu S, Liu H, Ma R. High TRIM44 expression in endometrial carcinoma is associated with a poorer patient outcome. Pathol Res Pract. 2018; 214: 727 31.

11. Jones HW. Treatment of adenocarcinoma of the endometrium. Obstet. Gynecol. Surv. 1975; 30: 147-69.

12. Creasman WT, Morrow CP, Bundy BN, Homesley HD, Graham JE, Heller PB. Surgical pathologic spread patterns of endometrial cancer. Cancer 1987; 60: 2035-41 PubMed.

13. Wolfson AH, Sightler SE, Markoe AM, Schwade JG, Averette HE, Ganjei P. The prognostic of surgical staging for carcinoma of the endometrium. Gynecol Oncol. 1992; 45: 142-6.

14. Morrow CP1, Bundy BN, Kurman RJ, Creasman WT, Heller P, Homesley HD. Relationship between surgical pathological risk factors and outcome in clinical stage I and II carcinoma of the endometrium A Gynecologic 
Oncology Group study. Gynecol Oncol. 1991; 40: 55-65. 15. Boronow RC, Morrow CP, Creasman WT, Disaia PJ, Silverberg SG, Miller A. Surgical staging in endometrial cancer: clinical-pathologic findings of a prospective study. Obstet Gynecol 1984; 63: 825-32.

16. Frei KA, Kinkel K, Bonél HM, Lu Y, Zaloudek C, Hricak H. Prediction of deep myometrial invasion in patients with endometrial cancer: clinical utility of contrast-enhanced MR imaging-a meta-analysis and Bayesian analysis. Radiology. 2000; 216: 444-9.

17. Lee KB, Ki KD, Lee JM, Lee JK, Kim JW, Cho CH. The risk of lymph node metastasis based on myometrial invasion and tumor grade in endometrioid uterine cancers: a multicenter, retrospective Korean study. Ann Surg Oncol. 2009; 16: 2882-7.

18. Sato S, Itamochi H, Shimada M, Fujii S, Naniwa J, Uegaki K. Preoperative and intraoperative assessments of depth of myometrial invasion in endometrial cancer. Int J Gynecol Cancer. 2009; 19: 884-7.

19. Soyi Lim, Kwang-Beom Lee, Chan-Yong Park. The prognostic significance of lymphovascular space involvement in patients with uterine confined endometrioid endometrial cancer. Korean J Obstet Gynecol 2012; 55: 76-82.

20. Briet JM, Hollema H, Reesink N, Aalders JG, Mourits MJ, ten Hoor KA. Lymphvascular space involvement: an independent prognostic factor in endometrial cancer. $G y$ necol Oncol 2005; 96: 799-804.

21. Ceccaroni M, Savelli L, Bovicelli A, Alboni C, Ceccarini M, Farina A. Prognostic value of pelvic lymphadenectomy in surgical treatment of apparent stage I endometrial cancer. Anticancer Res. 2004; 24(3b): 2073-8.

22. Ayhan A, Taskiran C, Celik C, Yuce K, Kucukali T. The influence of cytoreductive surgery on survival and morbidity in stage IVB endometrial cancer. Int J Gynecol Cancer 2002; 12: 448-53 PubMed . 